\title{
Coaching Educativo: Transformación conductual en estudiantes de bachillerato
}

\section{Educational Coaching: Behavioral Transformation in High School Students}

INFORMACIÓN DEL

\section{ARTÍCULO}

Fecha de recepción: 20 de Marzo de 2021.

Fecha de aceptación: 31 de Mayo de 2021.

Máster en Neuropsicología y Educación, Universidad Internacional de la Rioja. Docente - investigador, Universidad Espíritu Santo - Ecuador. E-mail: lalvarezsilva@uees.edu.ec Código ORCID

https://orcid.org/0000-0001-5835-714X

2 Máster en Neuropsicología y Educación, Universidad Internacional de la Rioja. Docente - investigador, Universidad Casa Grande - Ecuador. E-mail:

jahelelaine.gallegos@casagrande.edu.ec Código ORCID:

https://orcid.org/0000-0002-4610-9605

CITACIÓN: Álvarez Silva, L.A., \& Gallegos Miranda, J.E. (2021). Coaching Educativo: Transformación conductual en estudiantes de bachillerato. Podium, 39, 71-82. doi:10.31095/podium.2021.39.5

\section{ENLACE DOI:}

http://dx.doi.org/10.31095/podium.202 1.39 .5

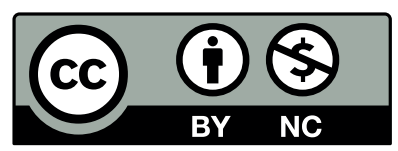

Luis Antonio Álvarez Silva ${ }^{1}$, Jahel Elaine Gallegos Miranda ${ }^{2}$

\section{Resumen}

El presente trabajo tiene como objetivo analizar el efecto que tiene el coaching educativo en el comportamiento del educando de bachillerato de una secundaria particular de Guayaquil. En este estudio se requirió de profesionales de la Psicología, certificados en coaching. La investigación presenta un enfoque cualitativo, con un diseño experimental de estudio de caso, de tipo longitudinal. Una de las herramientas utilizadas fue la META que permite lograr una adecuación de los objetivos del sujeto que la aplica. La muestra estuvo conformada por aquel educando que se encuentra en los tres últimos años de bachillerato; quienes tienen una edad promedio de 15 a 17 años y una baja significativa en sus índices conductuales. Lo resultados denotaron una mejora significativa en su conducta, optimizándose en un $20 \%$. Como conclusión se tiene que esta metodología, tiende a liberar el potencial del estudiante logrando una transformación positiva en su conducta.

\section{Palabras Clave:}

Educación, coaching educativo, comportamiento, estudiantes, academia, bachillerato.

Clasificación JEL: I21, D23, M12.

\begin{abstract}
This work aims to analyze the effect that educational coaching has on the behavior of high school students from a private high school in Guayaquil. This study required Psychology professionals, certified in Coaching. The research presents a qualitative approach, with an experimental design of a longitudinal case study. One of the tools used was the GOAL, which allows achieving an adaptation of objectives of the subject who applies it. The sample consisted of those students who are in the last three high school years; they are between 15/17 years old and have a significant drop in their behavioral indices. The outcome showed a significant behavioral improvement, optimized in $20 \%$. As a conclusion, this methodology tends to unleash the student's potential, achieving a positive behavioral transformation.
\end{abstract}

Keywords:

Education, educational coaching, behavior, students, academy, high school.

JEL Classification: I21, D23, M12.

$$
71
$$

PODIUM No. 39, Junio 2021, pp. 71-82

(C) Universidad Espíritu Santo - UEES

ISSN: 1390-5473 e-ISSN: 2588-0969 


\section{Introducción}

Es común, hoy por hoy, escuchar: ¡Voy a realizar un coaching! o ¡tengo mi sesión de coaching! Este término, que posee su origen etimológico en Europa, se encuentra revolucionando muchas secciones de la vida cotidiana del individuo, tales como las situaciones de índole personal, laboral, financiera, educativa, deportivo, interpersonal, intrapersonal, de liderazgo, motivacional, empresarial, cambiante, creativa, académica, entre otras (García-Naveira, 2013; Sans, 2012).

Este proceso tiene como meta, que el sujeto pueda alcanzar sus objetivos trazados en un tiempo determinado, dándole una puerta de salida a su situación actual versus su situación deseada. Sin embargo, para ello es indispensable que la persona que lleva a cabo el proceso conozca las herramientas necesarias para abordar la situación. Desde que uno de sus pioneros, Sir John Whitmore, presentara su obra Coaching: el método para mejorar el rendimiento de las personas (Whitmore, 2003), muchos entendidos de la temática propusieron una serie de libros al mercado mundial, dándole un mayor impacto, credibilidad, influencia.

Por otra parte, el comportamiento humano es una disciplina que se comenzó a estudiar desde el siglo IV mediante varios enunciados; uno de los que más resalta es la postura filosófica de la Mayéutica Socrática; esta corriente, resaltaba que, en un proceso de enseñanza, el alumno o discípulo descubra el conocimiento a través de la comunicación, el diálogo y la interrogación. Con el avance de los siglos, estos enunciados filosóficos fueron transformándose, aplicándose en ciencias y en procesos de gran incidencia: tal es el caso de la Educación, la Psicología, Pedagogía, entre otras. Siguiendo este orden de ideas, la educación es un proceso de gran incidencia en el desarrollo humano y en la sociedad. Esta se podría definir como el proceso de interacción entre dos o más sujetos que tiene como consecuencia la obtención de conocimientos, experticia, crecimiento ideológico, valores y comportamientos (Álvarez, Izquierdo, Gudiño y Macías, 2018).

Por esta razón, dentro de este proceso, los actores tienen un rol fundamental: el mismo que se orienta en el logro de la construcción de aquel conocimiento, basado en valores que beneficien a la sociedad y sobre todo llevando un nivel de comportamiento adecuado. Estos roles están enmarcados en profesiones que coadyuvan al logro de esta misión, tal como el personal docente $y$ de supervisión. Partiendo de este enunciado, la orientación del comportamiento del alumnado es elemental para el desarrollo académico. En consecuencia, múltiples investigaciones registradas en artículos científicos de base de datos de alto rigor académico, han hecho énfasis en abordar el coaching educativo (Baquero y Rodríguez, 2016; Bécart y Ramírez, 2016; Sánchez y Boronat, 2014), por lo que consideran que esta metodología proporciona cambios en el comportamiento del sujeto orientándolo 
en mejorar su diario actuar y desarrollo formativo. El presente estudio tiene como objetivo analizar el efecto que tiene el coaching educativo en el comportamiento de estudiantes de bachillerato de la entidad privada de la ciudad de Guayaquil. También se procedió a realizar un análisis teórico y cultural de temas educativos y comportamentales en el contexto mencionado, para luego cerrar con la etapa de las conclusiones.

\section{Revisión de literatura}

\section{Educación}

Etimológicamente, la palabra educación proviene del latín educare, término que se orienta a la crianza, guía y la promoción del desarrollo cognitivo de un individuo en determinado medio social. Se puede definir a la educación como aquel proceso que busca la formación integral de los sujetos desde una perspectiva cognitiva, potencial, psicológica, emocional, práctica, y/o espiritual con el fin de que éste pueda tener una vida equilibrada y pueda aportar positivamente a la sociedad, mediante su comportamiento (Souza, Posada y Lucio, 2019).

Esta definición de educación se alinea de forma contundente a la generación, creación y pulimento de conocimiento, el cual se objetiviza en la práctica en el denominado saber hacer; lo mismo que va desarrollando competencias en el individuo, las mismas que se las puede definir como aquellas características cognitivas y prácticas desarrolladas en el sujeto que le permiten pensar, actuar y comportarse de forma especializada ante determinada situación o contexto (Incháustegui, 2019).

Por tal razón, la educación de nivel básico y secundario se constituye en un aspecto predilecto en el que se crea, forma y potencializa el aprendizaje, el comportamiento y desarrollo humano a través de la labor de los educadores y personal responsable del comportamiento (Fuentes y Collado, 2019). S i $n$ embargo, la misma educación en reiteradas ocasiones ha sido objeto $\mathrm{y}$ blanco de críticas no constructivistas que la encasillan como un proceso que no provee lo que debe proveer, enmarcada por escenarios políticos, individualistas y mercantilistas (Biesta y Safstrom, 2018).

\section{Comportamiento}

La palabra comportamiento proviene del latín comportare, el mismo que puede dividirse en cum que significa: con; y portare, que deriva del verbo llevar. Es decir, es la forma de conllevarse, accionar o reaccionar frente a un determinado medio cultural y social. El comportamiento es una rama multidisciplinaria que se apega a muchas ciencias, tal es el caso del estudio del comportamiento del consumidor en el marketing (Encinas y Cavazos, 2016), el estudio del comportamiento de las abejas en la Apicultura (Vásquez, Mestanza y Alarcón, 2016), o el estudio del comportamiento organizacional en las empresas (Griffin, Phillips y Stanley, 2017). Sin embargo, la ciencia que ha aportado en mayor término al estudio de este aspecto es la Psicología, 
específicamente la Psicología norteamericana, denominada Psicología conductista. En efecto, según los psicólogos conductistas, el objeto de estudio de la misma es el comportamiento (Álvarez-Silva, Estrella-Marín y RosasBaldeón, 2018), en la que dicho comportamiento tiene características físicas, observables, y verificables, en efecto el ser vivo interactúa con el ambiente, y este con él (Galarsi, Medina, Ledezma y Zanin, 2011; Álvarez-Silva, 2018).

Tomando en consideración estas definiciones, se puede indicar que el sistema educativo en todas sus facetas, no está exento de presenciar modelos de comportamiento de todos sus actores sociales. Por lo tanto, siendo el alumnado un elemento de suma importancia en el proceso educativo, es de notable apreciación analizar donde este se desarrolla, interactúa y es condicionado por el entorno. El nivel de comportamiento de los alumnos de bachillerato presenta razones de análisis, tal es el caso de la vulnerabilidad a cambios comportamentales que presentan los adolescentes y jóvenes del nivel educativo de bachillerato, en algunos casos poseen un alto nivel de desorientación sobre el camino que van a tomar en su vida (Ramos, Sierra y Roces, 2019); provocando así que muchos de ellos continúen con los estudios superiores y otros no.

\section{Coaching educativo}

Originalmente, la palabra coach viene del inglés to coach que significa entrenar. Por tal razón, el término coaching, analizándolo desde una postura elemental, consistiría en un proceso de entrenamiento. Sin embargo, no es algo tan básico.

El termino coaching posee sus raíces etimológicas en el viejo continente y en Norteamérica; en sus inicios estaba orientado al entrenamiento deportivo; sin embargo, en las últimas décadas se ha potencializado en el ambito empresarial, consistiendo en una serie de actividades sincronizadas para que el directivo de determinada organización pueda desplegar su mayor potencial y liderazgo, reflejándose en el desarrollo de la compañía (Baquero y Rodríguez, 2016).

En sí, se puede definir el coaching como aquel proceso que permite al sujeto aumentar su potencial con el objetivo de mejorar su desempeño en cualquier ambito que éste se encuentre; sea este, mediante técnicas, comunicación asertiva, objetividad de metas y el reforzamiento del comportamiento (Álvarez et al., 2018). Por consiguiente, el coaching Educativo es una metodología que favorece el desarrollo del talento y comportamiento del estudiante, con el fin que éste progrese, logre metas, planifique su forma de ser, pensar y actuar (Sánchez y Boronat, 2014).

En el proceso de coaching Educativo, se puede considerar al profesor $\mathrm{o}$ inspector como coach y al estudiante como coachee. Sin embargo, es de suma importancia que la persona que gestiona el proceso tenga los conocimientos y práctica para poder ejecutar el procedimiento de forma grupal o en pareja. Uno de los mayores aportes a nivel de certificaciones en coaching se da 
en Reino Unido y España, estos países cuentan con carreras de grado $y$ postgrado en procesos coaching y sus avances son muy significativos (Ruiz, Boada, Merino y Ficapal, 2014).

En Ecuador, existen centros de nivel superior que permiten obtener certificaciones en coaching, con el fin de que la persona, mediante un riguroso proceso, aprenda y pueda gestionar procesos internos en empresas $\mathrm{y}$ entidades educativas particulares $\mathrm{o}$ estatales. En estas entidades se enseñan herramientas que permiten identificar información de los sujetos, tal es el caso del aprendizaje sobre la ventana de Johari, eficacia en comunicación asertiva, propuesta de metas y logro mediante indicadores y cuadros de gestión.

\section{Herramientas del coaching}

El coaching no se puede considerar ciencia o disciplina; de hecho, existen diferencias significativas a los campos de acción de ciencias establecidas como la Psicología, Neuropsicología, Psiquiatría, entre otras. Por otra parte, la perspectiva de todo libro con respecto al coaching tiene puntos en común y desacuerdos. Sin embargo, en su mayoría se presenta la definición de coaching utilizando como referencia a autores de alta membresía, tales como las analogías de Sócrates del siglo IV, Maquiavelo del siglo XV; y de épocas contemporáneas como Whitmore y Miedaner. Además, se considera que el coaching es un proceso medible y por ende utiliza herramientas de control (Whitmore, 2003; Alvarenga, Conceiçao, Viggiani, Moreno y Spina, 2019).
En el proceso, se pueden distinguir dos actores fundamentales, este es el coach (quien gestiona el proceso) y el coachee (quien recepta el proceso). Una de las herramientas básicas es la denominada M.E.T.A. que hace referencia a que cualquier objetivo del Coachee debe tener características medibles, específicas, tangibles y alcanzables. Otra de las herramientas utilizadas en la categoría básica es el diagrama DAFO, en el cual presenta un cuadrante que propone establecer cuáles son las debilidades, amenazas, fortalezas y oportunidades que tiene un determinado individuo ante un objetivo (Yuste, 2017).

\section{Metodología}

Tomando en consideración el aspecto académico, comportamental y la metodología del coaching Educativo, este estudio se enmarca bajo un enfoque cualitativo y un proceso de investigación experimental de tipo longitudinal; es decir fue aplicado en dos momentos determinados de tiempo, con características de estudio de caso y alcance descriptivo. En este se realizó un proceso de acompañamiento a jóvenes mediante el coaching educativo con el fin de analizar su nivel de comportamiento posterior a las sesiones grupales (comparando promedios conductuales entre trimestres).

El proceso de acompañamiento se realizó en el primer periodo de clases del 2019, que corresponde a los meses de mayo a agosto; y, en el segundo periodo académico del mismo año, el cual corresponde al rango de meses que van desde septiembre a diciembre. En este 
procedimiento se aplicó la observación técnica y una lista de cotejos conductuales como forma de registros de datos proporcionados por la entidad educativa. Con respecto al análisis estadístico, este no se presentó utilizando ningún programa de análisis en concordancia al estudio cualitativo, sin embargo, se aplicó un análisis descriptivo con base a tablas diseñadas en Excel. En sí, se procedió a utilizar los valores de los promedios de los resultados a nivel de conducta de los estudiantes.

Se aplicó un modelo de muestreo no probabilístico de tipo intencionado, el mismo que obedece al criterio de Hernández-Sampieri, Fernández y Baptista (2014). Para ello, el equipo investigador estuvo conformado por dos psicólogos certificados como Coachs por una entidad europea y con nexo a una universidad privada del Ecuador. El procedimiento coaching se realizó mediante la herramienta META y el diseño de objetivos SMART (específicos, medibles, alcanzables, realizables y temporales) de forma grupal y por segmentos.

El criterio para estructurar la muestra fue considerar los bajos promedios en conducta de los estudiantes que cursan los 3 últimos años de estudio de bachillerato, para lo cual se obtuvo una cantidad de 37 estudiantes (según los informes conductuales de la sección de bachillerato). La característica demográfica se la puede analizar en la Tabla 1.

Tabla 1.

Características de la muestra

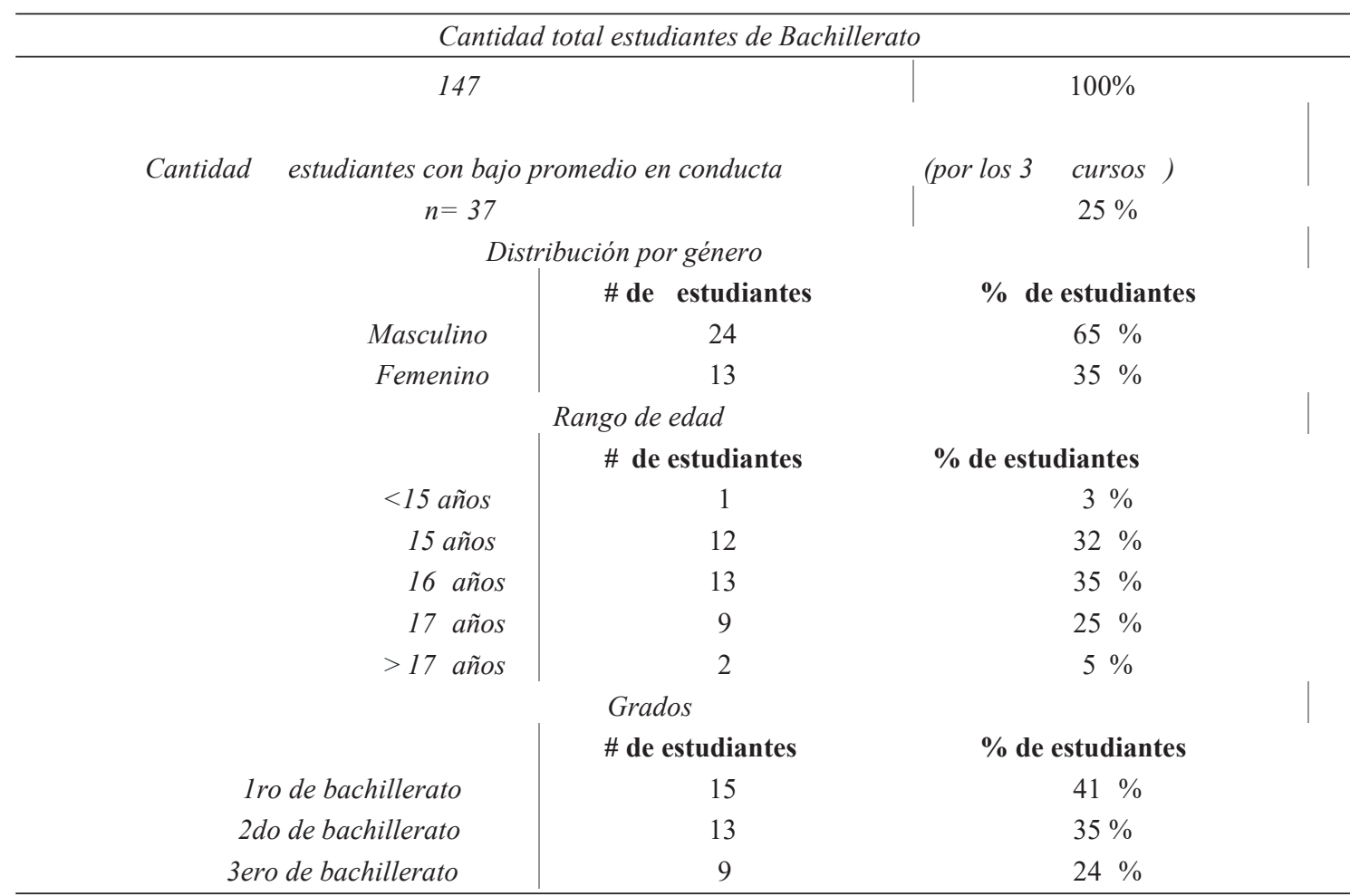

Fuente: Elaboración propia. 


\section{Resultados}

Desde la codificación de La Ley Orgánica de Educación Intercultural de Ecuador (Ministerio de Educación del Ecuador, 2017) que rige el sistema educativo del país hasta el grado de bachillerato, estableció que el comportamiento de un estudiante se debe medir por letras y no por números, es decir bajo un enfoque cualitativo. Es por ello que un estudiante que presenta un comportamiento en el que lidera sus responsabilidades en consonancia con la convivencia armónica, tiene una categoría de A (muy satisfactorio). El estudiante que solo cumple con las responsabilidades en relación a la sana convivencia, tiene una categoría de $\mathrm{B}$ (satisfactoria). El que falla de forma ocasional a sus responsabilidades dentro del marco de la armonía académica, tiene una distinción de $\mathrm{C}$ (poco satisfactoria). $\mathrm{Si}$ aquel estudiante tiene debilidades reiterativas en razón de su entorno, amerita la designación de D (Mejorable). Y, por último, si no cumple en lo absoluto con una relación de armonía con su entorno es designado con la categoría I (Insatisfactorio).

Tomando como referencia esta ley, no sería objetivo evaluar por medio de indicadores de gestión cuantitativos, comparando resultados variantes en el comportamiento del educando. Por tanto, y solo para el presente estudio. Estos resultados se presentan en las Tablas 2 y 3 , las cuales se obtuvieron en dos diferentes momentos de tiempo (antes de efectuar el proceso coaching y posteriormente).

En la Tabla 2 se puede notar un promedio referente a la obtención de información durante los primeros cinco meses de aquel año. Luego de efectuar el proceso coaching; se obtuvo una mejora del $20 \%$ al nivel comportamental, tal como lo muestra la Tabla 3.

Tabla 2.

Resultados del nivel de comportamiento en el educando de Bachillerato (1ro a 3ero) - 1er Periodo 2019

\begin{tabular}{lccccc}
\hline \multicolumn{1}{c}{$\mathbf{n}=\mathbf{3 7}$} & & Docente 1 & Docente 2 & Inspector & Promedio \\
\hline 1ro de bachillerato & 15 & $\overline{\mathrm{X}}: 4 / 10$ & $\overline{\mathrm{X}}: 6 / 10$ & $\overline{\mathrm{X}}: 5 / 10$ & $\overline{\mathrm{X}}: 5 / 10$ \\
2do de bachillerato & 13 & $\overline{\mathrm{X}}: 5 / 10$ & $\overline{\mathrm{X}}: 6 / 10$ & $\overline{\mathrm{X}}: 6 / 10$ & $\overline{\mathrm{X}}: 6 / 10$ \\
3ero de bachillerato & 9 & $\overline{\mathrm{X}}: 5 / 10$ & $\overline{\mathrm{X}}: 5 / 10$ & $\overline{\mathrm{X}}: 6 / 10$ & $\overline{\mathrm{X}}: 5 / 10$ \\
\hline
\end{tabular}

Global $\bar{X}: 5 / 10$

Fuente: Elaboración propia de los autores a partir de los datos proporcionados por el departamento de inspección, docencia y control.

Tabla 3

Resultados del nivel de comportamiento en el educando de Bachillerato (1 ro a 3ero) Después de efectuado el coaching Educativo - 2do Periodo 2019

\begin{tabular}{lccccc}
\hline \multicolumn{1}{c}{$\mathbf{n}=\mathbf{3 7}$} & & Docente 1 & Docente 2 & Inspector & Promedio \\
\hline 1ro de bachillerato & 15 & $\overline{\mathrm{x}}: 8 / 10$ & $\overline{\mathrm{x}}: 7 / 10$ & $\overline{\mathrm{x}}: 8 / 10$ & $\overline{\mathrm{x}}: 8 / 10$ \\
2do de bachillerato & 13 & $\overline{\mathrm{x}}: 7 / 10$ & $\overline{\mathrm{x}}: 7 / 10$ & $\overline{\mathrm{x}}: 7 / 10$ & $\overline{\mathrm{x}}: 7 / 10$ \\
3ero de bachillerato & 9 & $\overline{\mathrm{x}}: 8 / 10$ & $\overline{\mathrm{x}}: 7 / 10$ & $\overline{\mathrm{x}}: 7 / 10$ & $\overline{\mathrm{x}}: 7 / 10$ \\
\hline & & & & Global $\overline{\mathrm{x}}: \mathbf{7 / 1 0}$ \\
\hline
\end{tabular}

Fuente: Elaboración propia de los autores a partir de los datos proporcionados por el departamento de inspección, docencia y control. 
Además, tomando en consideración otros resultados de la aplicación del proceso en otros contextos, se tiene que el coaching educativo se puede aplicar a cualquiera de los agentes o actores sociales que forman parte del sistema educativo; sean estos estudiantes, padres, profesores y/o directivos; el mismo se orienta al desarrollo de las competencias involucrándose los pilares del saber, como se señalan evidencias de procesos de coaching en todos los niveles de entidades educativas de España (Bécart y Ramírez, 2016).

También, en un centro educativo de Colombia, en el área de biblioteca se procedió a implementar un nuevo sistema de gestión de calidad, en vista del alto nivel de resistencia al cambio por parte de los colaboradores, se procedió a utilizar la metodología de coaching; en sí, los resultados que se obtuvieron fueron de mucha utilidad y permitieron que los sujetos inmersos en ella, potencien sus capacidades y se orienten a una meta en común que dé como beneficio la consecución de la misión educativa (Rodríguez y Lombana, 2015).

Siguiendo este orden de ideas; en Norteamérica, específicamente en los Estados Unidos, el desarrollo de los procesos de coaching está sumamente avanzado, desde el ámbito de la primaria hasta el postgrado. Investigaciones en aquel país señalan que esta metodología es parte del desarrollo docente y estudiantil; y, en el proceso se utilizan talleres, grupos focales, reuniones, conversaciones asertivas significativas que ayudan al comportamiento de los adolescentes y les permite enfrentar dificultades educativas de mejor forma (Ramos et al., 2019).

Tomando como referencia Latinoamérica, la aplicación del coaching Educativo en una entidad secundaria privada de Trujillo - Perú, consistió en la realización de talleres a sus estudiantes para posteriormente realizar un análisis de su comportamiento mediante el Inventario de Adaptación de Conducta. En los resultados de esta investigación se evidenciaron cambios positivos en la conducta de los adolescentes por lo que se recomendó la continuidad del proceso coaching (Galloso, 2019).

En Ecuador, no existe una extensiva investigación sobre la temática; sin embargo, sí existe información en repositorios y bases de datos de prestigiosas Universidades del país, tales como la Universidad Espíritu Santo, Universidad Internacional del Ecuador, Universidad Católica de Cuenca, las mismas que dentro de su contenido tienen la implementación del coaching como una metodología que proporciona resultados positivos para el logro de metas personales, laborales y educativas (Urgilés, Erazo y Narvaez, 2019; Jiménez, Serrano, y Yance, 2017; Álvarez et al., 2018).

Como cierre de esta sección, un estudio en Ecuador sobre la implementación del coaching Educativo denota la elevación significativa del rendimiento académico, en este artículo la muestra fue de estudiantes jóvenes que 
no tienen mucho tiempo de haber terminado la secundaria; en esta información se evidencia la utilización de la ventana de Johari como herramienta de la metodología (Álvarez et al., 2018).

\section{Conclusiones}

El coaching Educativo es una metodología que lleva muchas décadas implementándose en lugares de alto desarrollo educativo; tales son los casos de Europa y Norteamérica. En el medio latinoamericano, se puede considerar como una corriente joven que da pasos agigantados. Existen en Ecuador investigaciones en tesis y artículos científicos que avalan el procedimiento con un efecto positivo en el comportamiento del educando, tal como se analizó en el presente estudio.

Es necesario que esta actividad se la realice con personas que conozcan de la temática, ya que conlleva bases profesionales de Psicología y un alto desarrollo de comunicación asertiva. Se puede definir al coaching Educativo como la acción de potencializar las competencias del estudiante, en cualquier ámbito que este se encuentre.

Esta modalidad, se puede aplicar desde la perspectiva docente $y$ de inspección, sin embargo el Coach no se puede autodenominar; ya que necesita conocer y aprender bases científicas y técnicas alineadas al comportamiento humano (Alvarez-Silva, 2018), las mismas que se adquieren en programas especializados o certificaciones en Coach, las cuales exigen un nivel de experiencia trabajando en grupos, liderando procesos y personas para posteriormente empezar un programa técnico que conlleva un tiempo determinado.

Las limitaciones de la aplicabilidad de esta metodología, giran en torno de conocer una herramienta que mida el efecto del coaching, lo cual es objeto de estudio por algún tiempo más. Sin embargo, el mejor comparativo es tener objetivos claros con indicadores de gestión que permitan analizar de forma objetiva la gestión del cambio.

Los preceptores y docentes de los ciclos de bachillerato y demás, pueden especializarse en la metodología de coaching Educativo con el fin de coadyuvar al moldear el comportamiento y desarrollo académico de los estudiantes, ya que esta metodología se orienta en resolver problemas, mejorar el rendimiento académico, mejorar la conducta, analizar y tomar mejores decisiones, escuchar activamente, comunicarse de forma asertiva y gestionar por medio de un adecuado liderazgo.

\section{Referencias}

Alvarenga, C., Conceiçao, C., Viggiani, S., Moreno, L., y Spina, E. (2019). A Janela de Johari como ferramenta de análise da privacidade de dados pessoais. Ciencia $d a$ información, 48(1), 79-93. Obtenido de http://revista.ibict.br/ciinf/article/downlo $\mathrm{ad} / 4250 / 4100 /$

Álvarez, L., Izquierdo, L., Gudiño, L., y Macías, M. (2018). Coaching educativo: desarrollo de competencias en el 
educando de nivel superior. INNOVA Research Journal, 3(11), 169-182. doi:https://doi.org/10.33890/innova.v3.n 11.2018.804

Álvarez-Silva, L. (2018). Relaciones Humanas laborales. PODIUM, (34), 89-92. doi:https://doi.org/10.31095/podium.201 8.34 .7

Álvarez-Silva, L., Estrella-Marín, B., y Rosas-Baldeón, M. (2018). Rol del Psicólogo Organizacional en la gestión de Talento. PODIUM, (33), 79-90. doi:https://doi. org/10.31095/podium.2018.33.8

Baquero, J., y Rodríguez, M. (2016). La relación entre el proceso de autorregulación y el proceso de coaching. Universitas Psychologica, 15(1), 15-25. doi:http://dx. doi.org/10.11144/Javeriana.upsy15-1.rpap

Bécart, A., y Ramírez, J. (2016). Fundamentos del coaching educativo: caracterización, aplicaciones y beneficios desde los cuatro pilares del saber. Plumilla Educativa, 18(2), 344-361. doi:http://dx.doi.org/10. 30554/plumillaedu.18.1973.2016

Biesta, G., y Safstrom, C. (2018). Un manifiesto por la educación. Praxis Educativa, 22(2), 20-36. doi:http://dx.doi.org/10.19 137/praxiseducativa-2018-220203

Encinas, F., y Cavazos, J. (2016). Validación de la escala de comportamiento ciudadano de consumidores de servicios educativos. Contaduría y Administración, 61(4), 649-665. doi:DOI: 10.1016/j.cya.2016.03.001

Fuentes, A., y Collado, J. (2019). Fundamentos epistemológicos transdisciplinares de educación y neurociencia. Sophia, Colección de Filosofía de la Educación(26), 83-113. doi:https://doi. org/10.17163/soph.n26.2019.02.

Galarsi, M., Medina, A., Ledezma, C., y Zanin, L. (2011). Comportamiento, historia y evolución.
Humanidades, XII(24), 89-123. Obtenido de http://fundamentos.unsl.edu.ar/pdf/ articulo-24-89.pdf

Galloso, S. (2019). Coaching educativo en la adaptación conductual de estudiantes de una institución educativa privada de Trujillo (Tesis de Postgrado). Trujillo: UCV. Obtenido de https:// repositorio.ucv.edu.pe/handle/20.500.126 $92 / 32772$

García-Naveira, A. (2013). Aplicación profesional del coaching en el deporte: un estudio de caso único. Cuadernos de Psicología del Deporte, 13(2), 101-112. Obtenido de https://revistas.um.es/cpd/article/view/18 0491/151351

Griffin, R., Phillips, J., y Stanley, G. (2017). Comportamiento organizacional, Administración de personas y organizaciones (12 ed.). México: Cengage Learning Editores S.A.

Hernández Sampieri, R., Fernández, C., y Baptista, M. (2014). Metodología de la Investigación (6 ed.). México: McGrawHill. Recuperado el 07 de 12 de 2020

Incháustegui, J. (2019). La base teórica de las competencias en educación. Educere, 23(74), 57-67. Obtenido de http:// epublica.saber.ula.ve/index.php/educere/ article/view/13803

Jiménez, S., Serrano, H., y Yance, C. (2017). Implementación de un programa de coaching para colaboradores de una PYME comercializadora de productos alimenticios. PODIUM, (27), 45-63. doi:https://doi.org/10.31095/podium.201 5.27 .3

Ministerio de Educaciòn del Ecuador. (2017). Ley organìca de educaciòn intercultural. Quito: Lexus.

Ramos, E., Sierra, B., y Roces, C. (2019). Ámbitos de aplicación del Coaching educativo: 
una revisión bibliográfica del periodo 2013-17. Educatio Siglo XXI, 37(2), 223-244. doi:https://doi.org/10.6018/ educatio. 387091

Rodríguez, L., y Lombana, A. (2015). Herramienta coaching como factor motivacional en la implementación de la NTC ISO 9001:2008 en el área de biblioteca de la Fundación Universitaria de Ciencias de la Salud (FUCS). SIGNOS-Investigación en Sistemas de Gestión, 7(2), 61-71. doi:https://doi.org/10.15332/s2145-1389. 2015.0002.04

Ruiz, M., Boada, J., Merino, E., y Ficapal, P. (2014). Una experiencia de coaching en estudiantes unversitarios. International Journal of Developmental and Educational Psychology, 4(1), 515-518. doi:https://doi.org/10.17060/ijodaep.201 4.n1.v4.638

Sánchez, B., y Boronat, J. (2014). Coaching educativo: modelo para el desarrollo de competencias intra e interpersonales. Educación XX1, 221-242. doi:https:// doi.org/10.5944/educxx1.17.1.10712

Sans, M. (2012). ¿Qué es el coaching? Sus Orígenes, definición, distintas metodologías $\mathrm{y}$ principios básicos de actuación de un coach. Revista de investigación 3Ciencias, 1-11. Obtenido de https://www.3ciencias.com/wp-content/u ploads/2012/06/3.Que-es-Coaching.pdf

Souza, M., Posada, S., y Lucio, P. (2019).

Neuroeducación: una propuesta pedagógica para la educación infantil. Revista Análisis, 51(94), 159-179. doi:https://doi.org/10.15332/s0120-8454. 2019.0094.08

Urgilés, L., Erazo, J., y Narvaez, C. (2019). El coaching y la productividad laboral en la Cooperativa de Ahorro y Crédito de la Pequeña Empresa Biblián Ltda. KOINONIA, 4(1), 408-435. doi: $10.35381 /$ r.k.v4i1.463
Vásquez, O., Mestanza, B., y Alarcón, R. (2016). Características morfométricas, comportamiento higiénico y agresividad de abejas criollas Apis mellifera sp. UCV-HACER. Revista de Investigación y Cultura, 5(1), 16-23. doi:https:// doi.org/10.18050/ucv-hacer.v5i1.956

Whitmore, J. (2003). Coaching: el método para mejorar el rendimiento de las personas. Barcelona: Paidos empresa.

Yuste, F. (2017). Herramientas de Coaching personal (4 ed.). Bilbao: Desclée de Brouwer. 
